\title{
Three-dimensional flow of an Oldroyd-B fluid over a bidirectional stretching surface with prescribed surface temperature and prescribed surface heat flux
}

\author{
Tasawar Hayat ${ }^{1,2}$, Sabir Ali Shehzad ${ }^{*}$, Saleh Al-Mezel ${ }^{2}$, Ahmed Alsaedi $^{2}$ \\ ${ }^{1}$ Department of Mathematics, Quaid-i-Azam University 45320, Islamabad 44000, Pakistan. \\ ${ }^{2}$ Department of Mathematics, Faculty of Science, King Abdulaziz University, P.O. Box 80257, Jeddah 21589, Saudi Arabia. \\ *Corresponding author. E-mail: ali_qau70@yahoo.com
}

\begin{abstract}
This paper concentrates on the mathematical modelling for three-dimensional flow of an incompressible Oldroyd-B fluid over a bidirectional stretching surface. Mathematical formulation incorporates the effect of internal heat source/sink. Two cases of heat transfer namely the prescribed surface temperature (PST) and prescribed surface heat flux (PHF) are considered. Computations for the governing nonlinear flow are presented using homotopy analysis method. Comparison of the present analysis is shown with the previous limiting result. The obtained results are discussed by plots of interesting parameters for both PST and PHF cases. We examine that an increase in Prandtl number leads to a reduction in PST and PHF. It is noted that both PST and PHF are increased with an increase in source parameter. Further we have seen that the temperature is an increasing function of ratio parameter.
\end{abstract}

Keywords: Three-dimensional flow; Oldroyd-B fluid; Bidirectional stretching surface; Heat source/sink.

\section{INTRODUCTION}

Heat transfer analysis over a stretched surface is a problem of great interest for the recent researchers. Such motivation is due to its various applications in the industrial and engineering processes like glass fiber and paper production, manufacture and drawing of plastics and rubber sheets, crystal growing, cooling of metallic sheets in a cooling bath, the continuous casting, glass blowing, polymer extrusion process, food processing, annealing and tinning of copper wires, heat treated materials travelling on conveyer belts and many others. Besides this, the concept of heat generation (source) or absorption (sink) is useful in applications involving heat removal from nuclear fuel debris, underground disposal of radioactive waste material, storage of food stuffs and dissociating fluids in packed-bed reactors. This concept is also important in view of problems, such as fluids undergoing exothermic/endothermic chemical reaction (Kandasamy et al., 2011) with the increasing demand of electronic technology, effective cooling of electronic equipment has become warranted. Such cooling of electronic equipment ranges from individual transistors to main frame computers and from energy suppliers to telephone switch boards (Kandasamy et al. (2005)).

There are many materials of multi-phase nature and of very high molecular weight involving in the chemical engineering, food stuff, bio-medicine and many others (Jamil and Fetecau, 2010). Such materials are called non-Newtonian fluids. The characteristics of non-Newtonian fluids are shear thinning, shear thickening, viscoelasticity, viscoplasticity. Such characteristics cannot be predicted by the Classical Navier-Stokes equations. For these fluids, there is a nonlinear relationship between the shear stress and shear rate. There is also not a single constitutive model which predicts all the characteristics of non-Newtonian fluids. Hence, different models of such fluids are introduced by the various investigators. An Oldroyd-B fluid is one subclass of the rate type non-Newtonian fluids which exhibit both relaxation and retardation times effects. Few studies in this direction may be represented by the refs. (Fetecau (2003), Fetecau and Fetecau (2005), Fetecau et al. (2007), Qi and Jin (2009), Tong et al. (2009), Zhang et al. (2011), Jamil et al. (2011), Jamil and Fetecau (2012), Zheng et al. (2012) and Hayat et al. (2012a)). Crane (1970) was the first who investigated the boundary layer two-dimensional flow of viscous fluid bounded by a stretching surface. Since then, different workers in the field have revisited similar problem through various physical aspects of heat and mass transfer, non-Newtonian fluids, magnetohydrodynamics, chemical reaction, suction, etc. However, majority of the existing studies relating to the titled problem has been examined for the two-dimensional flow (see few recent articles by Rashidi and Keimanesh (2010), Kazem et al. (2011), Hayat et al. (2011), Mukhopadhyay et al. (2011), Bhattacharyya (2012) and several refs. therein). Very limited attention is given to the three-dimensional flow over a stretching surface. For example, Liu and Andersson (2008) studied the three-dimensional flow of viscous fluid induced by a bidirectional surface in the presence of variable thermal conditions. Flow of viscous fluid generated by the bidirectional stretching sheet was numerically discussed by Wang (1984). Ariel (2007) presented a study for three-dimensional flow of viscous fluid over a linear stretching surface. Ahmed et al. (2011) carried out a study for MHD three-dimensional flow of viscous fluid in a porous medium. Heat transfer characteristics are taken in the presence of prescribed surface temperature and prescribed surface heat flux. Very recently, Shehzad et al. (2012) studied the three-dimensional flow of Jeffery fluid with convective surface conditions.

To our knowledge, no analysis is presented to investigate the three-dimensional flow of non-Newtonian fluid over a bidirectional stretching surface with heat transfer. Even such flow analysis in absence of heat transfer is not available so far. Hence, constitutive equations of Oldroyd-B fluid are employed in the mathematical formulation. The stretching surface exhibits the heat transfer through two cases namely PST and PHF. Effects of heat generation/absorption are also present. Computations for governing problem are made using homotopy analysis method (HAM) (Liao (2003), Rashidi and Domairry (2009), Rashidi and Pour (2010), Turkyilmazoglu (2010), Keimanesh et al. (2011), Rashidi et al. (2011), Vosughi et al. (2011), and Hayat et al. (2012b, 2012c, 2012d). The obtained series solu- 
tions are examined in detail for convergence and physical interpretation.

\section{FLOW MODELS}

Consider three-dimensional boundary layer flow of an incompressible Oldroyd-B fluid. The flow is induced by bidirectional stretching surface (at $z=0$ with prescribed surface temperature (PST) and prescribed surface heat flux (PHF) (see Fig. 1). Steady flow of an incompressible fluid is considered for $z>$ 0 . Flow analysis is carried out in the presence of heat source/sink. The conservation equations of mass, momentum and energy for steady flow can be expressed as

$\operatorname{div} \mathbf{V}=\mathbf{0}$,

$\rho \frac{d \mathbf{V}}{d t}=\operatorname{div} \mathbf{T}$

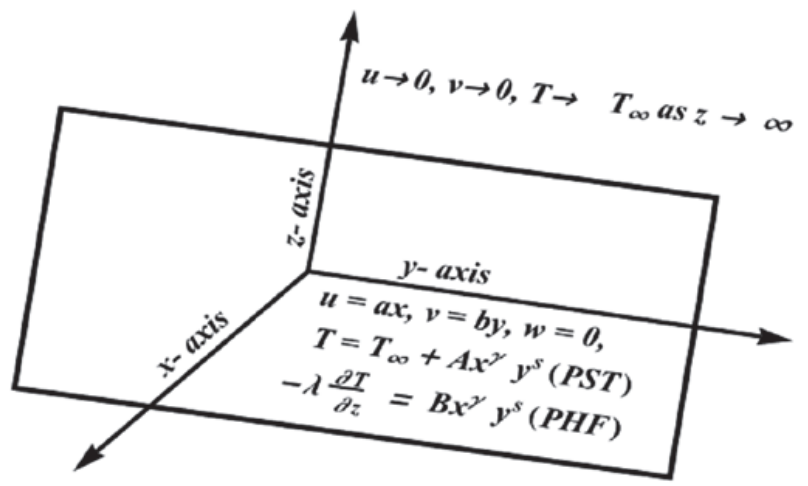

Fig. 1. Physical model.

The Cauchy stress tensor $\mathbf{T}$ and extra stress tensor $\mathbf{S}$ in the above equation are defined as:

$\mathbf{T}=-p \mathbf{I}+\mathbf{S}$

$\mathbf{S}+\lambda_{1} \frac{D \mathbf{S}}{D t}=\mu\left(\mathbf{A}_{1}+\lambda_{2} \frac{D \mathbf{A}_{1}}{D t}\right)$,

$(\mathbf{V} . \nabla) \mathbf{T}=\sigma \nabla^{2} \mathbf{T}+q\left(T-T_{\infty}\right)$,

where $D / D t$ is the Covariant differentiation, and $\lambda_{1}$ and $\lambda_{2}$ are the relaxation time and retardation time, respectively. The first Rivlin Ericksen tensor $\mathbf{A}_{1}$ is defined as

$\mathbf{A}_{1}=\operatorname{grad} V+(\operatorname{grad} V)^{*}$,

where $*$ indicates the matrix transpose and the velocity field $\mathbf{V}$ is taken as following

$\mathbf{V}=[u(x, y, z), v(x, y, z), w(x, y, z)]$.

The definition of $D / D t$ is (Harris, 1977):

$\frac{D a_{i}}{D t}=\frac{\partial a_{i}}{\partial t}+u_{r} a_{i, r}-u_{i, r} a_{r}$

Following the procedure of Harris (1977) at pages 221-223, Eqs. (1)-(5) now give: $\frac{\partial u}{\partial x}+\frac{\partial v}{\partial y}+\frac{\partial w}{\partial z}=0$

$$
\begin{aligned}
& u \frac{\partial u}{\partial x}+v \frac{\partial u}{\partial y}+w \frac{\partial u}{\partial z}+ \\
& \lambda_{1}\left(\begin{array}{c}
u^{2} \frac{\partial^{2} u}{\partial x^{2}}+v^{2} \frac{\partial^{2} u}{\partial y^{2}}+w^{2} \frac{\partial^{2} u}{\partial z^{2}}+2 u v \frac{\partial^{2} u}{\partial x \partial y} \\
+2 v w \frac{\partial^{2} u}{\partial y \partial z}+2 u w \frac{\partial^{2} u}{\partial x \partial z}
\end{array}\right) \\
& \left.=-\frac{\partial p}{\partial x}+v\left(\begin{array}{c}
\frac{\partial^{2} u}{\partial x^{2}}+\frac{\partial^{2} u}{\partial y^{2}}+\frac{\partial^{2} u}{\partial z^{2}}+ \\
u \frac{\partial^{3} u}{\partial x^{3}}+u \frac{\partial^{3} u}{\partial x \partial y^{2}}+u \frac{\partial^{3} u}{\partial x \partial z^{2}} \\
+v \frac{\partial^{3} u}{\partial x^{2} \partial y}+u \frac{\partial^{3} u}{\partial y^{3}}+v \frac{\partial^{3} u}{\partial y \partial z^{2}}+w \frac{\partial^{3} u}{\partial x^{2} \partial z} \\
+w \frac{\partial^{3} u}{\partial y^{2} \partial z}+w \frac{\partial^{3} u}{\partial z^{3}}-\frac{\partial u}{\partial x} \frac{\partial^{2} u}{\partial x^{2}}-\frac{\partial u}{\partial x} \frac{\partial^{2} u}{\partial y^{2}} \\
-\frac{\partial u}{\partial x} \frac{\partial^{2} u}{\partial z^{2}}-\frac{\partial u}{\partial y} \frac{\partial^{2} v}{\partial x^{2}}-\frac{\partial u}{\partial y} \frac{\partial^{2} v}{\partial y^{2}}-\frac{\partial u}{\partial y} \frac{\partial^{2} v}{\partial z^{2}} \\
-\frac{\partial u}{\partial z} \frac{\partial^{2} w}{\partial x^{2}}-\frac{\partial u}{\partial z} \frac{\partial^{2} w}{\partial y^{2}}-\frac{\partial u}{\partial z} \frac{\partial^{2} w}{\partial z^{2}}
\end{array}\right)\right)
\end{aligned}
$$

$$
\begin{gathered}
u \frac{\partial v}{\partial x}+v \frac{\partial v}{\partial y}+w \frac{\partial v}{\partial z}+ \\
\lambda_{\mathrm{I}}\left(\begin{array}{c}
u^{2} \frac{\partial^{2} v}{\partial x^{2}}+v^{2} \frac{\partial^{2} v}{\partial y^{2}}+w^{2} \frac{\partial^{2} v}{\partial z^{2}}+2 u v \frac{\partial^{2} v}{\partial x \partial y} \\
+2 v w \frac{\partial^{2} v}{\partial y \partial z}+2 u w \frac{\partial^{2} v}{\partial x \partial z}
\end{array}\right) \\
=-\frac{\partial p}{\partial y}+v\left(\begin{array}{c}
\frac{\partial^{2} v}{\partial x^{2}}+\frac{\partial^{2} v}{\partial y^{2}}+\frac{\partial^{2} v}{\partial z^{2}}+ \\
u \frac{\partial^{3} v}{\partial x^{3}}+u \frac{\partial^{3} v}{\partial x \partial y^{2}}+u \frac{\partial^{3} v}{\partial x \partial z^{2}} \\
\lambda_{2}\left(\begin{array}{c}
+v \frac{\partial^{3} v}{\partial x^{2} \partial y}+v \frac{\partial^{3} v}{\partial y^{3}}+v \frac{\partial^{3} v}{\partial y \partial z^{2}}+w \frac{\partial^{3} v}{\partial x^{2} \partial z} \\
+w \frac{\partial^{3} v}{\partial y^{2} \partial z}+w \frac{\partial^{3} v}{\partial z^{3}}-\frac{\partial v}{\partial x} \frac{\partial^{2} u}{\partial x^{2}}-\frac{\partial v}{\partial x} \frac{\partial^{2} u}{\partial y^{2}} \\
-\frac{\partial v}{\partial x} \frac{\partial^{2} u}{\partial z^{2}}-\frac{\partial v}{\partial y} \frac{\partial^{2} v}{\partial x^{2}}-\frac{\partial v}{\partial y} \frac{\partial^{2} v}{\partial y^{2}}-\frac{\partial v}{\partial y} \frac{\partial^{2} v}{\partial z^{2}} \\
-\frac{\partial v}{\partial z} \frac{\partial^{2} w}{\partial x^{2}}-\frac{\partial v}{\partial z} \frac{\partial^{2} w}{\partial y^{2}}-\frac{\partial v}{\partial z} \frac{\partial^{2} w}{\partial z^{2}}
\end{array}\right)
\end{array}\right),
\end{gathered}
$$

$$
u \frac{\partial w}{\partial x}+v \frac{\partial w}{\partial y}+w \frac{\partial w}{\partial z}+
$$$$
\lambda_{\mathrm{I}}\left(\begin{array}{c}
u^{2} \frac{\partial^{2} w}{\partial x^{2}}+v^{2} \frac{\partial^{2} w}{\partial y^{2}}+w^{2} \frac{\partial^{2} w}{\partial z^{2}}+2 u v \frac{\partial^{2} w}{\partial x \partial y} \\
+2 v w \frac{\partial^{2} w}{\partial y \partial z}+2 u w \frac{\partial^{2} w}{\partial x \partial z}
\end{array}\right)
$$

$$
\left.=-\frac{\partial p}{\partial z}+v\left(\begin{array}{c}
\frac{\partial^{2} w}{\partial x^{2}}+\frac{\partial^{2} w}{\partial y^{2}}+\frac{\partial^{2} w}{\partial z^{2}}+ \\
u \frac{\partial^{3} w}{\partial x^{3}}+u \frac{\partial^{3} w}{\partial x \partial y^{2}}+u \frac{\partial^{3} w}{\partial x z^{2}} \\
+v \frac{\partial^{3} w}{\partial x^{2} \partial y}+v \frac{\partial^{3} w}{\partial y^{3}}+v \frac{\partial^{3} w}{\partial y \partial z^{2}}+w \frac{\partial^{3} w}{\partial x^{2} \partial z} \\
+w \frac{\partial^{3} w}{\partial y^{2} \partial z}+w \frac{\partial^{3} w}{\partial z^{3}}-\frac{\partial w}{\partial x} \frac{\partial^{2} u}{\partial x^{2}}-\frac{\partial w}{\partial x} \frac{\partial^{2} u}{\partial y^{2}} \\
-\frac{\partial w}{\partial x} \frac{\partial^{2} u}{\partial z^{2}}-\frac{\partial w}{\partial y} \frac{\partial^{2} v}{\partial x^{2}}-\frac{\partial w}{\partial y} \frac{\partial^{2} v}{\partial y^{2}}-\frac{\partial w}{\partial y} \frac{\partial^{2} v}{\partial z^{2}} \\
-\frac{\partial w}{\partial z} \frac{\partial^{2} w}{\partial x^{2}}-\frac{\partial w}{\partial z} \frac{\partial^{2} w}{\partial y^{2}}-\frac{\partial w}{\partial z} \frac{\partial^{2} w}{\partial z^{2}}
\end{array}\right)\right)
$$

After neglecting the pressure gradient and using the standard boundary layer assumptions (Schichting, 1964), the resulting equations for three-dimensional flow of an Oldroyd-B fluid with heat transfer are 


$$
\begin{aligned}
& \frac{\partial u}{\partial x}+\frac{\partial v}{\partial y}+\frac{\partial w}{\partial z}=0 \\
& u \frac{\partial u}{\partial x}+v \frac{\partial u}{\partial y}+w \frac{\partial u}{\partial z}+ \\
& \lambda_{1}\left(\begin{array}{c}
u^{2} \frac{\partial^{2} u}{\partial x^{2}}+v^{2} \frac{\partial^{2} u}{\partial y^{2}}+w^{2} \frac{\partial^{2} u}{\partial z^{2}}+2 u v \frac{\partial^{2} u}{\partial x \partial y} \\
+2 v w \frac{\partial^{2} u}{\partial y \partial z}+2 u w \frac{\partial^{2} u}{\partial x \partial z}
\end{array}\right) \\
& =v\left(\frac{\partial^{2} u}{\partial z^{2}}+\lambda_{2}\left(\begin{array}{c}
u \frac{\partial^{3} u}{\partial x \partial z^{2}}+v \frac{\partial^{3} u}{\partial y \partial z^{2}}+ \\
w \frac{\partial^{3} u}{\partial z^{3}}-\frac{\partial u}{\partial x} \frac{\partial^{2} u}{\partial z^{2}} \\
-\frac{\partial u}{\partial y} \frac{\partial^{2} v}{\partial z^{2}}-\frac{\partial u}{\partial z} \frac{\partial^{2} w}{\partial z^{2}}
\end{array}\right)\right), \\
& u \frac{\partial v}{\partial x}+v \frac{\partial v}{\partial y}+w \frac{\partial v}{\partial z}+ \\
& \lambda_{1}\left(\begin{array}{c}
u^{2} \frac{\partial^{2} v}{\partial x^{2}}+v^{2} \frac{\partial^{2} v}{\partial y^{2}}+w^{2} \frac{\partial^{2} v}{\partial z^{2}}+2 u v \frac{\partial^{2} v}{\partial x \partial y}+ \\
2 v w \frac{\partial^{2} v}{\partial y \partial z}+2 u w \frac{\partial^{2} v}{\partial x \partial z}
\end{array}\right) \\
& =v\left(\frac{\partial^{2} v}{\partial z^{2}}+\lambda_{2}\left(\begin{array}{c}
u \frac{\partial^{3} v}{\partial x \partial z^{2}}+v \frac{\partial^{3} v}{\partial y \partial z^{2}} \\
+w \frac{\partial^{3} v}{\partial z^{3}}-\frac{\partial v}{\partial x} \frac{\partial^{2} v}{\partial z^{2}} \\
-\frac{\partial v}{\partial y} \frac{\partial^{2} v}{\partial z^{2}}-\frac{\partial v}{\partial z} \frac{\partial^{2} w}{\partial z^{2}}
\end{array}\right)\right), \\
& u \frac{\partial T}{\partial x}+v \frac{\partial T}{\partial y}+w \frac{\partial T}{\partial z}=k_{1} \frac{\partial^{2} T}{\partial z^{2}}+\frac{q}{\rho c_{p}}\left(T-T_{\infty}\right)
\end{aligned}
$$

Note that in the above equations, the boundary layer approximations are used. Further, $u, v$ and $w$ are the velocity components in the $x-, y$ - and $z$-directions, $T$ the fluid temperature, $k_{1}$ the thermal diffusivity of the fluid, $v=(\mu / \rho)$ the kinematic viscosity, $\rho$ the density of fluid, $\mu$ the dynamic viscosity of fluid, $c_{p}$ the specific heat at constant pressure of the fluid and $q$ is the heat source/sink parameter with $q>0$ (heat source) and $q<0$ (heat sink). [20]:

The associated boundary conditions are defined as follows

$$
\begin{aligned}
& u=u_{w}(x)=a x, v=v_{w}(y)=b y, w=0 \text { at } z=0, \\
& u \rightarrow 0, v \rightarrow 0 \text { as } z \rightarrow \infty
\end{aligned}
$$

For temperature, the corresponding boundary conditions are given by:

Type I. Prescribed surface temperature (PST):

$$
\begin{aligned}
T & =T_{w}(x, y)=T_{\infty}+A x^{\gamma} y^{s} \text { at } z=0, \\
T & \rightarrow T_{\infty} \text { as } z \rightarrow \infty .
\end{aligned}
$$

Type II. Prescribed surface heat flux (PHF):

$$
\begin{aligned}
&-\lambda \frac{\partial T}{\partial z}=B x^{\gamma} y^{s} \text { at } z=0, \\
& T \rightarrow T_{\infty} \text { as } z \rightarrow \infty .
\end{aligned}
$$

Here, $\lambda$ is the thermal conductivity of the fluid, $T_{\infty}$ the constant temperature outside the thermal boundary layer, $A$ and $\mathrm{B}$ the positive constants. The power indices $\gamma$ and $s$ determine how the temperature or the heat flux varies in $x y$ - plane.

Considering Ahmad et al. (2011):

$$
\begin{gathered}
u=a x f^{\prime}(\eta), v=a y g^{\prime}(\eta), \eta=z \sqrt{\frac{a}{v}}, \\
w=-\sqrt{a v}(f(\eta)+g(\eta)), \\
\operatorname{PST}: \theta(\eta)=\frac{T(x, y, z)-T_{\infty}}{T_{w}(x, y)-T_{\infty}} \\
\operatorname{PHF}: T(x, y, z)-T_{\infty}=\frac{B}{\lambda} \sqrt{\frac{v}{a}} x^{\gamma} y^{s} \phi(\eta)
\end{gathered}
$$

Eq. (13) is automatically satisfied and Eqs. (14)-(17) take the following forms:

$$
\begin{aligned}
& f^{\prime \prime \prime}+(f+g) f^{\prime \prime}-f^{\prime 2}+\beta_{1}\left(\begin{array}{c}
2(f+g) f^{\prime} f^{\prime \prime}- \\
(f+g)^{2} f^{\prime \prime \prime}
\end{array}\right) \\
& +\beta_{2}\left(\left(f^{\prime \prime}+g^{\prime \prime}\right) f^{\prime \prime}-(f+g) f^{\prime \prime \prime \prime}\right)=0, \\
& g^{\prime \prime \prime}+(f+g) g^{\prime \prime}-g^{2}+\beta_{1}\left(\begin{array}{c}
2(f+g) g^{\prime} g^{\prime \prime} \\
-(f+g)^{2} g^{\prime \prime \prime}
\end{array}\right) \\
& +\beta_{2}\left(\left(f^{\prime \prime}+g^{\prime \prime}\right) g^{\prime \prime}-(f+g) g^{\prime \prime \prime \prime}\right)=0, \\
& \theta^{\prime \prime}+\operatorname{Pr}(f+g) \theta^{\prime}+\operatorname{Pr}\left(\beta-\gamma f^{\prime}-s g^{\prime}\right) \theta=0, \\
& \phi^{\prime \prime}+\operatorname{Pr}(f+g) \phi^{\prime}+\operatorname{Pr}\left(\beta-\gamma f^{\prime}-s g^{\prime}\right) \phi=0, \\
& f=0, \quad g=0, f^{\prime}=1, g^{\prime}=\alpha, \\
& \theta=1, \phi^{\prime}=-1 \text { at } \eta=0, \\
& f^{\prime} \rightarrow 0, g^{\prime} \rightarrow 0, \theta \rightarrow 0, \phi \rightarrow 0 \text { as } \eta \rightarrow \infty,
\end{aligned}
$$

where $\beta_{1}=\lambda_{1} a$ and $\beta_{2}=\lambda_{2} a$ are the Deborah numbers, $\alpha=\frac{b}{a}$ the ratio of stretching rates, $\operatorname{Pr}=\frac{v}{k_{1}}$ the Prandtl number, $k_{1}$ the thermal diffusivity and $\beta=\frac{q}{\rho a c_{p}}$ the internal heat parameter.

\section{HOMOTOPY ANALYSIS SOLUTIONS}

In this section, we solve the problem consisting of Eqs. (21)-(25) by HAM. For that the initial guesses and auxiliary linear operators are taken as follows:

$$
\begin{aligned}
& f_{0}(\eta)=\left(1-e^{-\eta}\right), g_{0}(\eta)=\beta\left(1-e^{-\eta}\right), \\
& \theta_{0}(\eta)=\exp (-\eta), \phi_{0}(\eta)=\exp (-\eta),
\end{aligned}
$$




$$
\begin{aligned}
& L(f)=f^{\prime \prime \prime}-f^{\prime}, L(g)=g^{\prime \prime \prime}-g^{\prime}, \\
& L(\theta)=\theta^{\prime \prime}-\theta, L(\phi)=\phi^{\prime \prime}-\phi .
\end{aligned}
$$

Subject to the properties

$$
\begin{aligned}
L(f)\left(C_{1}+C_{2} e^{\eta}+C_{3} e^{-\eta}\right) & =0, \\
L(g)\left(C_{4}+C_{5} e^{\eta}+C_{6} e^{-\eta}\right) & =0, \\
L(\theta)\left(C_{7} e^{\eta}+C_{8} e^{-\eta}\right) & =0, \\
L(\phi)\left(C_{9} e^{\eta}+C_{10} e^{-\eta}\right) & =0,
\end{aligned}
$$

where $C_{i} \quad(i=1-10)$ are the arbitrary constants.

At zeroth order, the problems satisfy:

$$
(1-q) L_{f}\left(\hat{f}(\eta ; q)-f_{0}(\eta)\right)
$$

$=q \hbar_{f} \mathbf{N}_{f}(\hat{f}(\eta ; q), \hat{g}(\eta ; q))$,

$$
(1-q) L_{g}\left(\hat{g}(\eta ; q)-g_{0}(\eta)\right)
$$$$
=q \hbar_{g} \mathbf{N}_{g}(\hat{f}(\eta ; q), \hat{g}(\eta ; q)) \text {, }
$$

$$
(1-q) L_{\theta}\left(\hat{\theta}(\eta ; q)-\theta_{0}(\eta)\right)
$$

$=q \hbar_{\theta} \mathbf{N}_{\theta}(\hat{f}(\eta ; q), \hat{g}(\eta ; q), \hat{\theta}(\eta ; q))$,

$$
(1-q) L_{\phi}\left(\hat{\phi}(\eta ; q)-\phi_{0}(\eta)\right)
$$$$
=q \hbar_{\phi} \mathbf{N}_{\phi}(\hat{f}(\eta ; p), \hat{g}(\eta ; q), \hat{\phi}(\eta ; q)) \text {, }
$$

$$
\hat{f}(0 ; q)=0, \hat{f}^{\prime}(0 ; q)=1, \hat{f}^{\prime}(\infty ; q)=0,
$$$$
\hat{g}(0 ; q)=0, \hat{g}^{\prime}(0 ; q)=\alpha, \hat{g}^{\prime}(\infty ; q)=0 \text {, }
$$$$
\hat{\theta}(0 ; q)=1, \hat{\theta}(\infty, q)=0 \text {, }
$$$$
\hat{\phi}^{\prime}(0, q)=0 \hat{\phi}(\infty, q)=0 \text {, }
$$

$\mathbf{N}_{f}[\hat{f}(\eta, q), \hat{g}(\eta, q)]=\frac{\partial^{3} \hat{f}(\eta, q)}{\partial \eta^{3}}-\left(\frac{\partial \hat{f}(\eta, q)}{\partial \eta}\right)^{2}$

$+(\hat{f}(\eta, q)+\hat{g}(\eta, q)) \frac{\partial^{2} \hat{f}(\eta, q)}{\partial \eta^{2}}$

$+\beta_{1}\left(\begin{array}{c}2(\hat{f}(\eta, q)+\hat{g}(\eta, q)) \frac{\partial \hat{f}(\eta, q)}{\partial \eta} \frac{\partial^{2} \hat{f}(\eta, q)}{\partial \eta^{2}} \\ -(\hat{f}(\eta, q)+\hat{g}(\eta, q))^{2} \frac{\partial^{3} f(\eta, q)}{\partial \eta^{2}}\end{array}\right)$

$+\beta_{2}\left(\begin{array}{c}\left(\frac{\partial^{2} \hat{f}(\eta, q)}{\partial \eta^{2}}+\frac{\partial^{2} \hat{g}(\eta, q)}{\partial \eta^{2}}\right) \frac{\partial^{2} \hat{f}(\eta, q)}{\partial \eta^{2}} \\ -(\hat{f}(\eta, q)+\hat{g}(\eta, q)) \frac{\partial^{4} \hat{f}(\eta, q)}{\partial \eta^{4}}\end{array}\right)$,

$\mathbf{N}_{g}[\hat{g}(\eta, q), \hat{f}(\eta, q)]=\frac{\partial^{3} \hat{g}(\eta, q)}{\partial \eta^{3}}-\left(\frac{\partial \hat{g}(\eta, q)}{\partial \eta}\right)^{2}$

$+(\hat{f}(\eta, q)+\hat{g}(\eta, q)) \frac{\partial^{2} \hat{g}(\eta, q)}{\partial \eta^{2}}$

$+\beta_{1}\left(\begin{array}{c}2(\hat{f}(\eta, q)+\hat{g}(\eta, q)) \frac{\partial \hat{g}(\eta, q)}{\partial \eta} \frac{\partial^{2} \hat{g}(\eta, q)}{\partial \eta^{2}} \\ -(\hat{f}(\eta, q)+\hat{g}(\eta, q))^{2} \frac{\partial^{3} \hat{g}(\eta, q)}{\partial \eta^{2}}\end{array}\right)$

$+\beta_{2}\left(\begin{array}{c}\left(\frac{\partial^{2} \hat{f}(\eta, q)}{\partial \eta^{2}}+\frac{\partial^{2} \hat{g}(\eta, q)}{\partial \eta^{2}}\right) \frac{\partial^{2} \hat{g}(\eta, q)}{\partial \eta^{2}} \\ -(\hat{f}(\eta, q)+\hat{g}(\eta, q)) \frac{\partial^{4} \hat{g}(\eta, q)}{\partial \eta^{4}}\end{array}\right)$,
$\mathbf{N}_{\theta}[\hat{\theta}(\eta, q), \hat{f}(\eta, q), \hat{g}(\eta, q)]=\frac{\partial^{2} \hat{\theta}(\eta, q)}{\partial \eta^{2}}$

$+\operatorname{Pr}(\hat{f}(\eta, q)+\hat{g}(\eta, q)) \frac{\partial \hat{\theta}(\eta, q)}{\partial \eta}$

$+\operatorname{Pr}\left(\begin{array}{c}\beta-\gamma \frac{\partial \hat{f}(\eta, q)}{\partial \eta} \\ -s \frac{\partial \hat{g}(\eta, q)}{\partial \eta}\end{array}\right) \hat{\theta}(\eta, q)$,

$\mathbf{N}_{\phi}[\hat{\phi}(\eta, q), \hat{f}(\eta, q), \hat{g}(\eta, q)]=\frac{\partial^{2} \hat{\phi}(\eta, q)}{\partial \eta^{2}}$

$+\operatorname{Pr}(\hat{f}(\eta, q)+\hat{g}(\eta, q)) \frac{\partial \hat{\phi}(\eta, q)}{\partial \eta}$

$+\operatorname{Pr}\left(\begin{array}{c}\beta-\gamma \frac{\partial \hat{f}(\eta, q)}{\partial \eta} \\ -s \frac{\partial \hat{g}(\eta, q)}{\partial \eta}\end{array}\right) \hat{\phi}(\eta, q)$.

In above expressions, $q$ shows the embedding parameter, $\hbar_{f}, \hbar_{g}, \hbar_{\theta}$ and $\hbar_{\phi}$ the non-zero auxiliary parameters and $\mathbf{N}_{f}, \mathbf{N}_{g}, \mathbf{N}_{\theta}$ and $\mathbf{N}_{\phi}$ the nonlinear operators. When $q=0$ and $q=1$ then we obtain

$$
\begin{gathered}
\hat{f}(\eta ; 0)=f_{0}(\eta), \hat{g}(\eta, 0)=g_{0}(\eta), \\
\hat{\theta}(\eta, 0)=\theta_{0}(\eta), \hat{\phi}(\eta, 0)=\phi_{0}(\eta) \\
\hat{f}(\eta ; 1)=f(\eta), \hat{g}(\eta, 1)=g(\eta), \\
\hat{\theta}(\eta, 1)=\theta(\eta), \hat{\phi}(\eta, 1)=\phi(\eta) .
\end{gathered}
$$

It should be pointed out that when $q$ increases from 0 to 1 , the $f(\eta, q), g(\eta, q)$ and $\theta(\eta, q)$ vary from $f_{0}(\eta)$, $g_{0}(\eta), \theta_{0}(\eta), \phi_{0}(\eta)$ to $f(\eta), g(\eta), \theta(\eta)$ and $\phi(\eta)$. Using Taylors' expansion we write:

$$
\begin{aligned}
& f(\eta, q)=f_{0}(\eta)+\sum_{m=1}^{\infty} f_{m}(\eta) q^{m}, \\
& g(\eta, q)=g_{0}(\eta)+\sum_{m=1}^{\infty} g_{m}(\eta) q^{m}, \\
& \theta(\eta, q)=\theta_{0}(\eta)+\sum_{m=1}^{\infty} \theta_{m}(\eta) q^{m}, \\
& \phi(\eta, q)=\phi_{0}(\eta)+\sum_{m=1}^{\infty} \phi_{m}(\eta) q^{m}, \\
& f_{m}(\eta)=\left.\frac{1}{m !} \frac{\partial^{m} f(\eta ; q)}{\partial \eta^{m}}\right|_{q=0}, \\
& g_{m}(\eta)=\left.\frac{1}{m !} \frac{\partial^{m} g(\eta ; q)}{\partial \eta^{m}}\right|_{q=0}, \\
& \theta_{m}(\eta)=\left.\frac{1}{m !} \frac{\partial^{m} \theta(\eta ; q)}{\partial \eta^{m}}\right|_{q=0}, \\
& \phi_{m}(\eta)=\left.\frac{1}{m !} \frac{\partial^{m} \phi(\eta ; q)}{\partial \eta^{m}}\right|_{q=0},
\end{aligned}
$$

where the parameters $\hbar_{f}, \hbar_{g}, \hbar_{\theta}$ and $\hbar_{\phi}$ have a key role in the convergence of series solutions. The values of parameters 
are chosen in such a manner that Eqs. (39)-(42) converge at $q=$ 1. Hence Eqs. (39)-(42) give:

$$
\begin{aligned}
& f(\eta)=f_{0}(\eta)+\sum_{m=1}^{\infty} f_{m}(\eta), \\
& g(\eta)=g_{0}(\eta)+\sum_{m=1}^{\infty} g_{m}(\eta), \\
& \theta(\eta)=\theta_{0}(\eta)+\sum_{m=1}^{\infty} \theta_{m}(\eta), \\
& \phi(\eta)=\phi_{0}(\eta)+\sum_{m=1}^{\infty} \phi_{m}(\eta) .
\end{aligned}
$$

The general solutions are arranged as follows:

$$
\begin{aligned}
& f_{m}(\eta)=f_{m}^{*}(\eta)+C_{1}+C_{2} e^{\eta}+C_{3} e^{-\eta} \\
& g_{m}(\eta)=g_{m}^{*}(\eta)+C_{4}+C_{5} e^{\eta}+C_{6} e^{-\eta} \\
& \theta_{m}(\eta)=\theta_{m}^{*}(\eta)+C_{7} e^{\eta}+C_{8} e^{-\eta} \\
& \phi_{m}(\eta)=\phi_{m}^{*}(\eta)+C_{9} e^{\eta}+C_{10} e^{-\eta}
\end{aligned}
$$

in which the special solutions are denoted by $f_{m}^{*}, g_{m}^{*}, \theta_{m}^{*}$ and $\phi_{m}^{*}$.

\section{CONVERGENCE OF THE HOMOTOPY SOLUTIONS}

We know that the homotopy analysis method provides a great freedom to select the auxiliary parameters $\hbar_{f}, \hbar_{g}, \hbar_{\theta}$ and $\hbar_{\phi}$ regarding adjustment and control of the convergence of series solutions. To determine the appropriate convergence interval of the constructed series solutions, the $\hbar-$ curves at 17 th-order of approximations are sketched. Figs. $2-5$ supports that the range of admissible values of $\hbar_{f}, \hbar_{g}, \hbar_{\theta}$ and $\hbar_{\phi}$ are $-0.95 \leq \hbar_{f} \leq-0.2,-0.85 \leq \hbar_{g} \leq-0.15, \quad-1.25 \leq \hbar_{\theta} \leq-0.6$ and $-1.1 \leq \hbar_{\phi} \leq-0.45$.

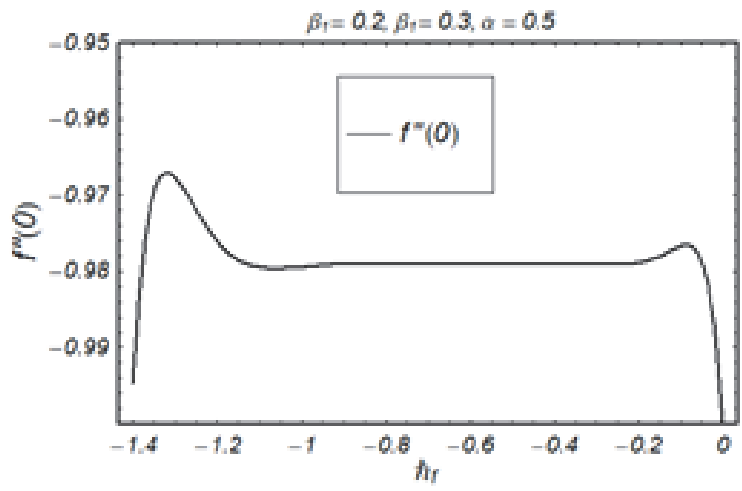

Fig. 2. $\hbar$-curve for the function $f(\eta)$.

\section{GRAPHICAL RESULTS AND DISCUSSION}

The graphical illustrations of temperature field corresponding to various interesting quantities for prescribed surface tem- perature (PST) and prescribed surface heat flux (PHF) are visualized in this section. Figs. $6-12$ present the variations of $\beta_{1}, \beta_{2}$, $\gamma, \beta, \operatorname{Pr}, \alpha$ and $s$ on the prescribed surface temperature $\theta(\eta)$ and prescribed surface heat flux $\phi(\eta)$.

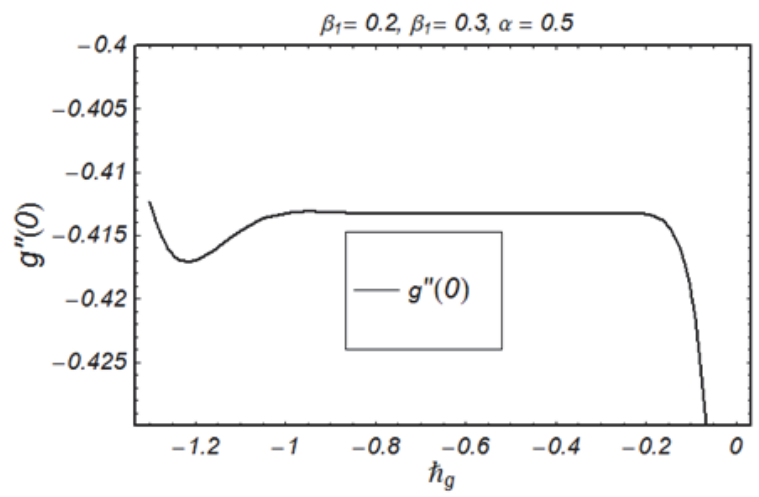

Fig. 3. $\hbar$-curve for the function $g(\eta)$.

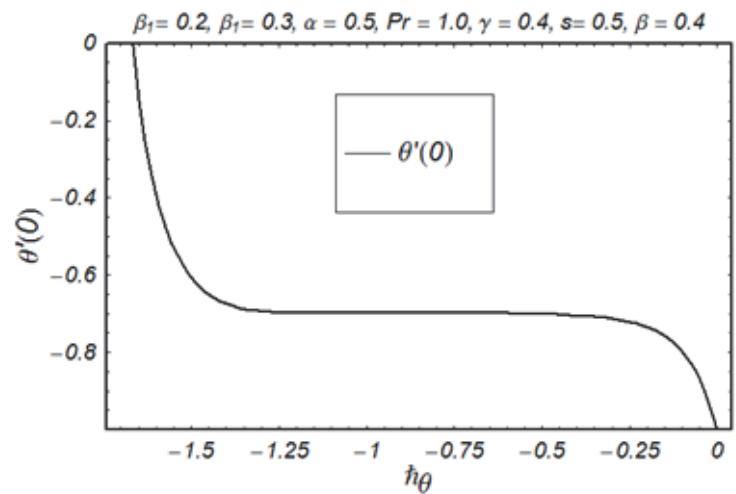

Fig. 4. $\hbar$-curve for the function $\theta(\eta)$.

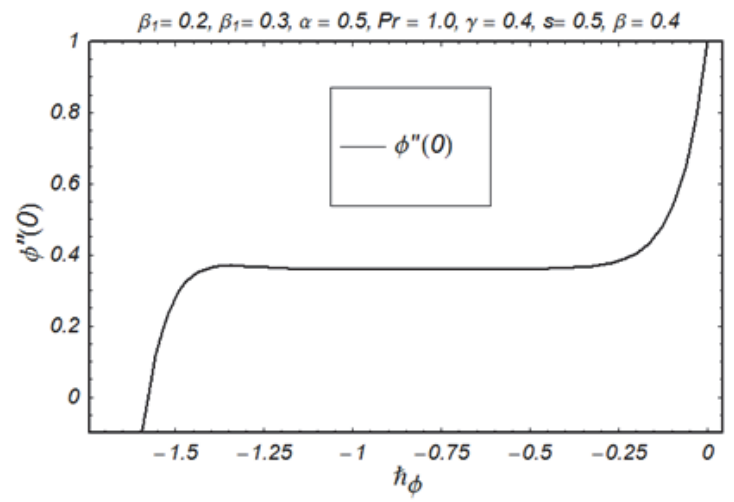

Fig. 5. $\hbar$-curve for the function $\phi(\eta)$.

We observe from Fig. $6(\mathrm{a}, \mathrm{b})$ that the temperature profiles $\theta(\eta), \phi(\eta)$ and their associated thermal boundary layer thicknesses are increased when Deborah $\beta_{1}$ increases. As expected that an increase in the Deborah number $\beta_{1}$ leads to an increase in relaxation time. When relaxation time increases then it give rise to the temperature fields $\theta(\eta)$ and $\phi(\eta)$ The variations of $\beta_{2}$ on $\theta(\eta)$ and $\phi(\eta)$ are seen in Fig. $7(\mathrm{a}, \mathrm{b})$. Here it is found that the temperature profiles $\theta(\eta)$ and $\phi(\eta)$ are decreasing functions of $\beta_{2}$. An increase in Deborah number $\beta_{2}$ is due to 
Table 1. Convergence analysis of series solutions by numerical data for different order of deformations when $\beta_{1}=0.2, \beta_{2}=0.3$, $\alpha=s=0.5, \quad \operatorname{Pr}=1.0, \quad \gamma=\beta=0.4, \quad \hbar_{f}=\hbar_{g}=0.6 \quad$ and $\hbar_{\theta}=\hbar_{\phi}=-0.9$.

\begin{tabular}{lcccc}
$\begin{array}{l}\text { Order of } \\
\text { deformations }\end{array}$ & $-f^{\prime \prime}(0)$ & $-g^{\prime \prime}(0)$ & $\theta^{\prime}(0)$ & $\phi^{\prime \prime}(0)$ \\
\hline 1 & 0.967500 & 0.42625 & 0.79000 & 0.53500 \\
10 & 0.979098 & 0.41324 & 0.69988 & 0.35889 \\
15 & 0.979101 & 0.41328 & 0.69744 & 0.35888 \\
20 & 0.979108 & 0.41328 & 0.69669 & 0.35897 \\
25 & 0.979107 & 0.41328 & 0.69642 & 0.35901 \\
30 & 0.979107 & 0.41328 & 0.69637 & 0.35901 \\
35 & 0.979107 & 0.41328 & 0.69637 & 0.35901 \\
40 & 0.979107 & 0.41328 & 0.69637 & 0.35901 \\
\hline
\end{tabular}

Table 2. Numerical values of $f^{\prime \prime}(0), g^{\prime \prime}(0), f(\infty)$ and $g(\infty)$. for different values of $\alpha$ when $\beta_{1}=\lambda_{1}=0$.

\begin{tabular}{lccccc}
\hline & $\alpha$ & $f^{\prime \prime}(0)$ & $g^{\prime \prime}(0)$ & $f(\infty)$ & $g(\infty)$ \\
\hline Wang (1984) & 0.0 & -1 & 0 & 1 & 0 \\
Present & & -1 & 0 & 1 & 0 \\
\hline Wang (1984) & 0.25 & -1.048813 & -0.194564 & 0.907075 & 0.257986 \\
Present & & -1.04881 & -0.19457 & 0.907047 & 0.25790 \\
\hline Wang (1984) & 0.50 & -1.093097 & -0.465205 & 0.842360 & 0.451671 \\
Present & & -1.09309 & -0.46522 & 0.84293 & 0.45169 \\
\hline Wang (1984) & 0.75 & -1.134485 & -0.794622 & 0.792308 & 0.612049 \\
Present & & -1.13450 & -0.79462 & 0.79231 & 0.61214 \\
\hline Wang (1984) & 1.0 & -1.173720 & -1.173720 & 0.751527 & 0.751527 \\
Wang (1984) & & -1.17372 & -1.17372 & 0.75149 & 0.75149 \\
\hline
\end{tabular}

Table 3. Temperature gradient $\theta^{\prime}(0)$ for different values of $\alpha, \gamma$ and $s$ with $\beta_{1}=\beta_{2}=\beta=0.0$ and $\operatorname{Pr}=1.0$.

\begin{tabular}{|c|c|c|c|c|c|c|}
\hline & & $\gamma=s=0$ & $\gamma=-2, s=0$ & $\gamma=2, s=0$ & $\gamma=0, s=-2$ & $\gamma=0, s=2$ \\
\hline $\begin{array}{l}\text { Liu and } \\
\text { Andersson } \\
\text { (2008) }\end{array}$ & $\alpha=0.25$ & -0.665933 & 0.554512 & -1.364890 & -0.413111 & -0.883125 \\
\hline Present & & -0.66593 & 0.55457 & -1.36489 & -0.41310 & -0.88312 \\
\hline $\begin{array}{l}\text { Liu and } \\
\text { Andersson } \\
\text { (2008) }\end{array}$ & $\alpha=0.50$ & -0.735334 & 0.308578 & -1.395356 & -0.263381 & -1.106491 \\
\hline Present & & -0.73533 & 0.30858 & -1.39536 & -0.26338 & -1.10649 \\
\hline $\begin{array}{l}\text { Liu and } \\
\text { Andersson } \\
\text { (2008) }\end{array}$ & $\alpha=0.50$ & -0.796472 & 0.135471 & -1.425038 & -0.126679 & -1.292003 \\
\hline Present & & -0.79472 & 0.13547 & -1.42504 & -0.12667 & -1.29200 \\
\hline
\end{tabular}

a)

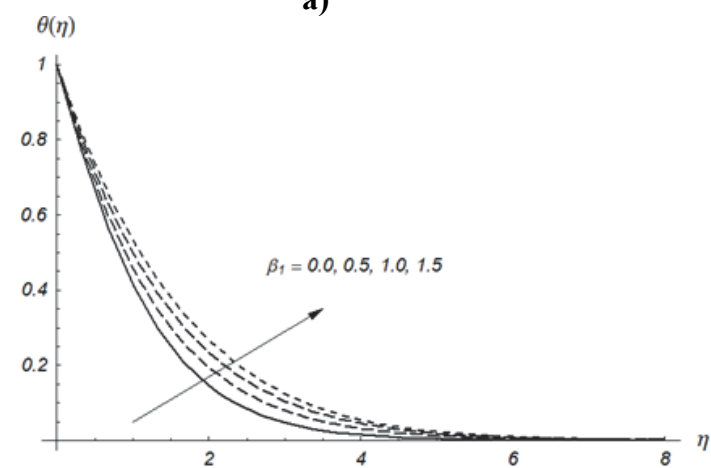

b)

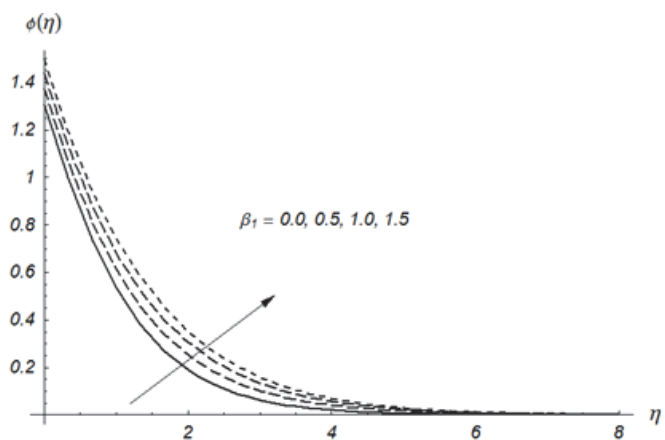

Fig. 6 (a, b). Temperature profiles $\theta(\eta)$ and $\phi(\eta)$ for various values of Deborah number $\beta_{1}$ when $\beta_{2}=0.3, \operatorname{Pr}=1.0, \alpha=s=0.5$ and $\gamma=\beta=0.4$. 

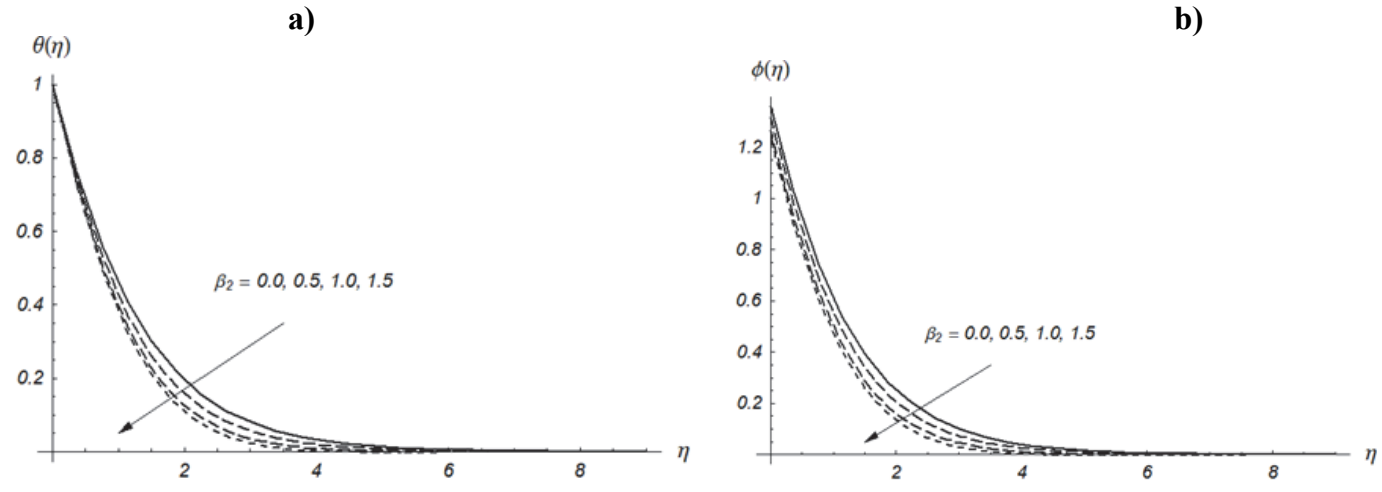

Fig. 7 (a, b). Temperature profiles $\theta(\eta)$ and $\phi(\eta)$ for various values of Deborah number $\beta_{2}$ when $\beta_{1}=0.3, \operatorname{Pr}=1.0, \alpha=s=0.5$ and $\gamma=\beta=0.4$.
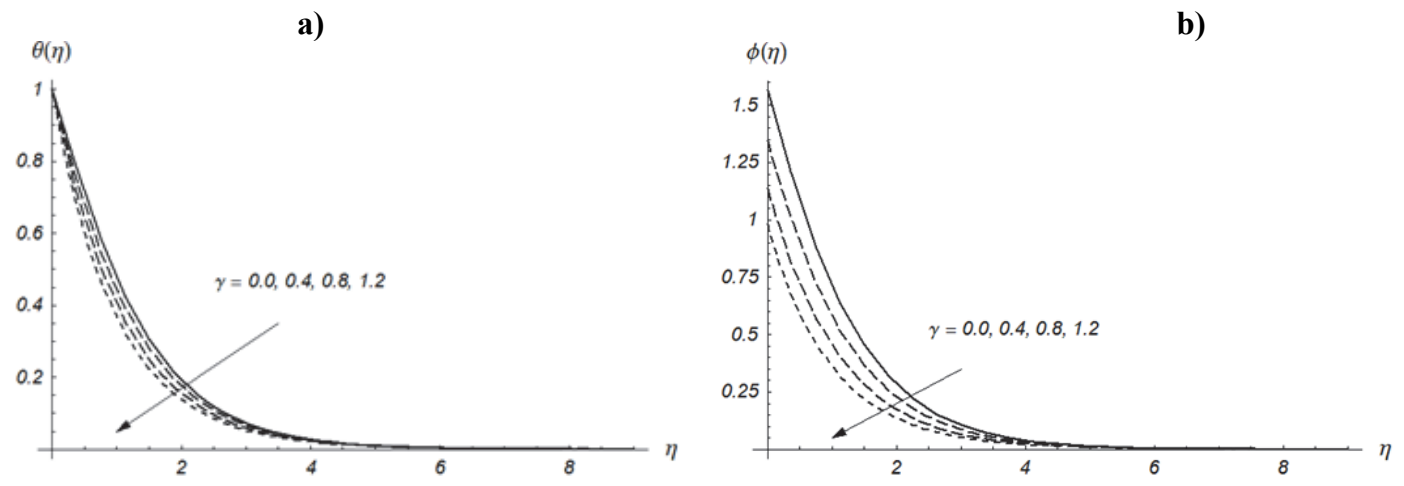

Fig. $8(\mathbf{a}, \mathbf{b})$. Temperature profiles $\theta(\eta)$ and $\phi(\eta)$ for various values of $\gamma$ when $\beta_{1}=\beta_{2}=0.3, \operatorname{Pr}=1.0, \alpha=s=0.5$ and $\beta=0.4$.
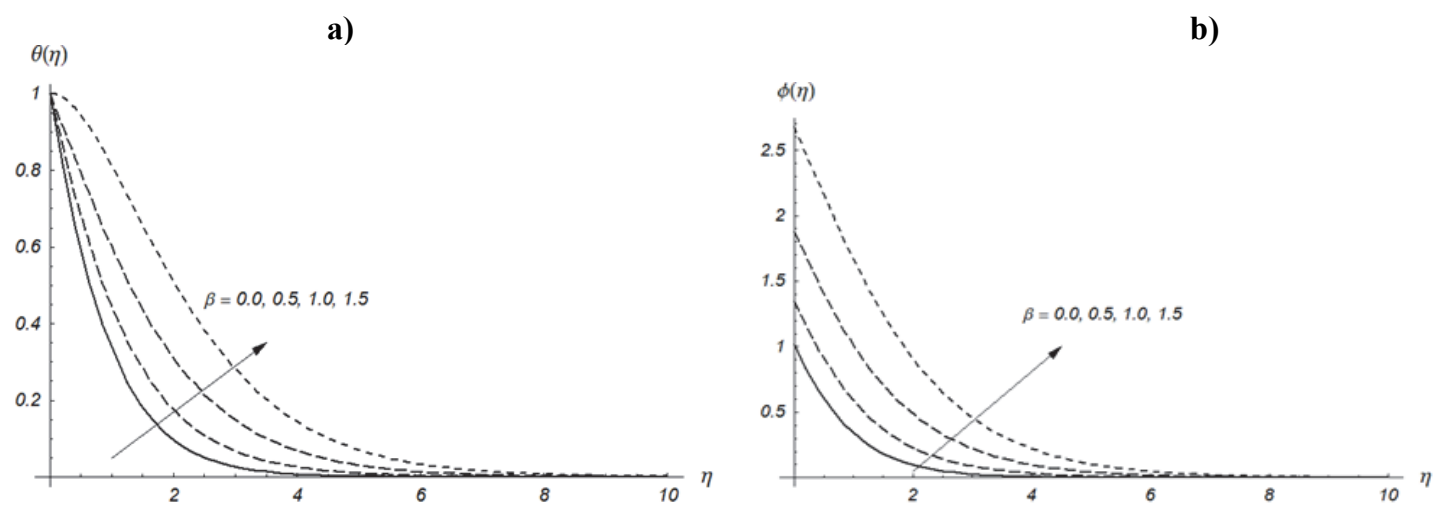

Fig. 9 (a, b). Temperature profiles $\theta(\eta)$ and $\phi(\eta)$ for various values of $\beta$ when $\beta_{1}=\beta_{2}=0.3, \operatorname{Pr}=1.0, \alpha=s=0.5$ and $\gamma=0.4$.

a)

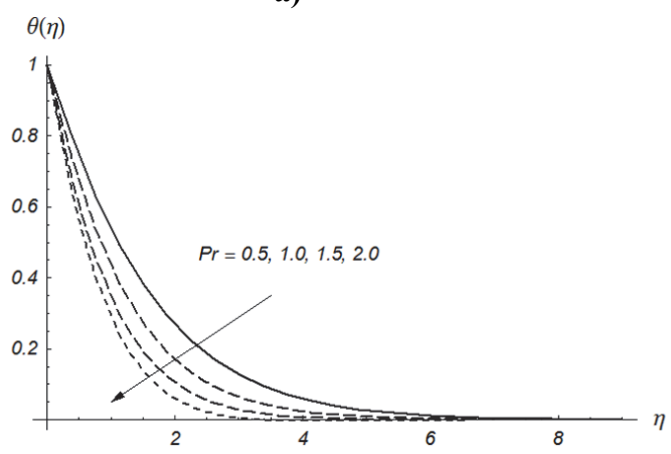

b)

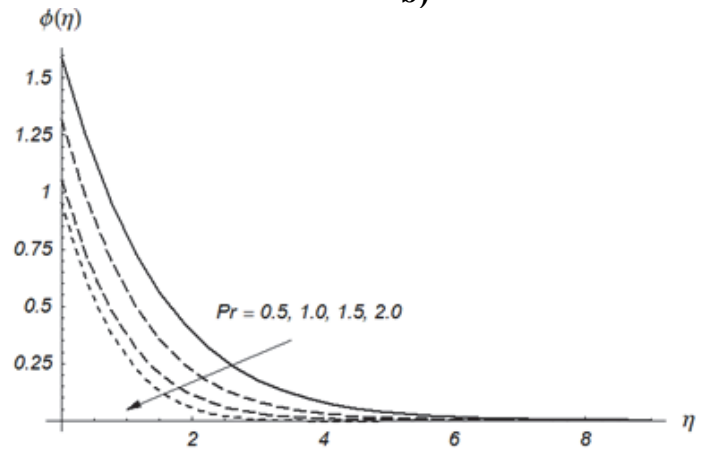

Fig. 10 (a, b). Temperature profiles $\theta(\eta)$ and $\phi(\eta)$ for various values of $\operatorname{Pr}$ when $\beta_{1}=\beta_{2}=0.3, \alpha=s=0.5$ and $\beta=\gamma=0.4$. 
a)

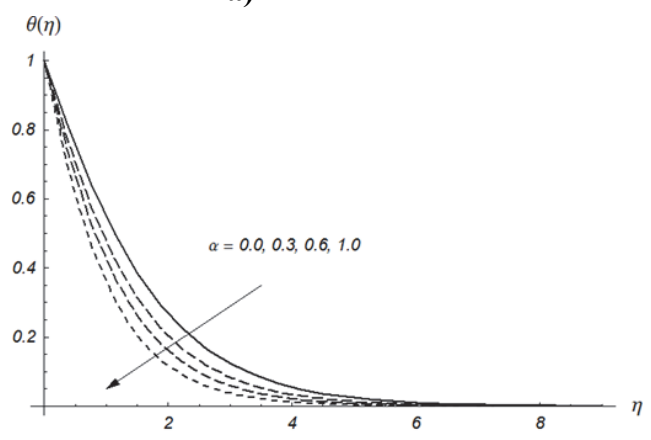

b)

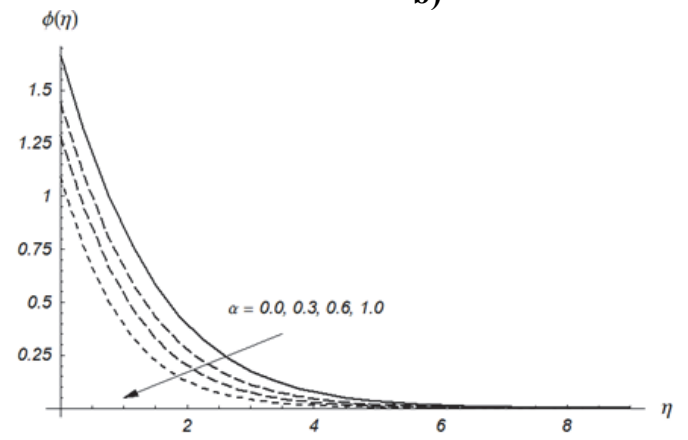

Fig. $11(\mathbf{a}, \mathbf{b})$. Temperature profiles $\theta(\eta)$ and $\phi(\eta)$ for various values of $\alpha$ when $\beta_{1}=\beta_{2}=0.3, \operatorname{Pr}=1.0, s=0.5$ and $\beta=\gamma=0.4$.

a)

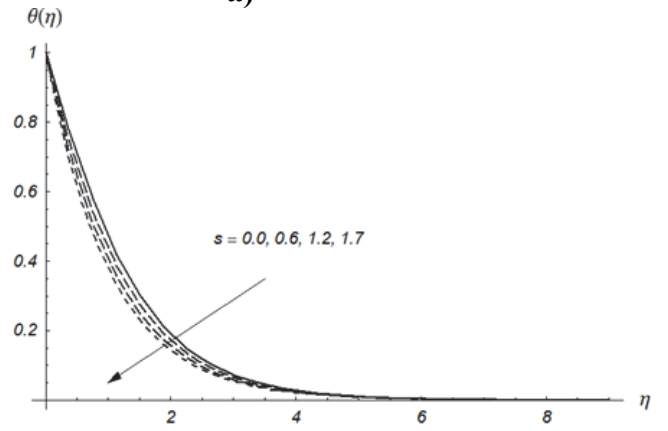

b)

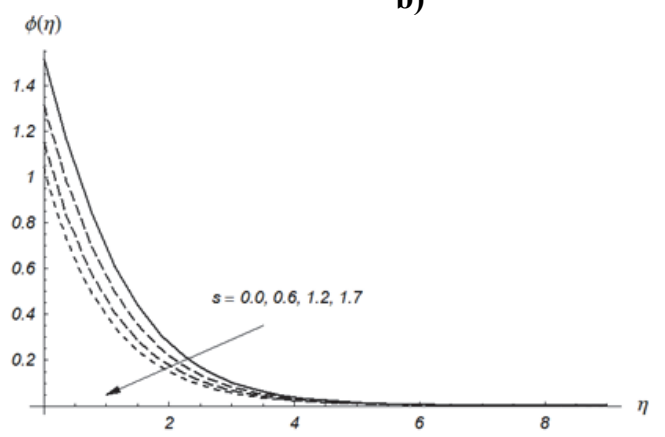

Fig. $12(\mathbf{a}, \mathbf{b})$. Temperature profiles $\theta(\eta)$ and $\phi(\eta)$ for various values of $s$ when $\beta_{1}=\beta_{2}=0.3, \operatorname{Pr}=1.0, \alpha=0.5$ and $\beta=\gamma=0.4$.

increase in retardation time. There is a decrease in $\theta(\eta)$ and $\phi(\eta)$ when retardation time increases. A comparison of Figs. 6 $(\mathrm{a}, \mathrm{b})$ and $7(\mathrm{a}, \mathrm{b})$ shows that Deborah number $\beta_{1}$ and $\beta_{2}$ have quite opposite effects on the temperature profiles $\theta(\eta)$ and $\phi(\eta)$. An increase in $\gamma$ creates a reduction in temperature profiles $\theta(\eta)$ and $\phi(\eta)$. From Fig. $8(\mathrm{a}, \mathrm{b})$ it can be examined that $\gamma$ have similar effects on $\theta(\eta)$ and $\phi(\eta)$ but the variations in $\phi(\eta)$ are large in comparison to $\theta(\eta)$. Also we analyzed that the temperature $\phi(\eta)$ at the wall is decreased when $\gamma$ increases. The effects of heat source/sink parameter $\beta$ on $\theta(\eta)$ and $\phi(\eta)$ are illustrated in Fig. $9(\mathrm{a}, \mathrm{b})$. It is found that there is an increase in $\theta(\eta), \phi(\eta)$ and their associated thermal boundary thicknesses with an increase in $\beta$. Fig. $10(\mathrm{a}, \mathrm{b})$ presents the variations of Prandtl number $\operatorname{Pr}$ on $\theta(\eta)$ and $\phi(\eta)$. The temperature profiles $\theta(\eta), \phi(\eta)$ and thermal boundary layer thickness are reduced when Prandtl number is increased. In fact an increase in Prandtl number reduces the thermal diffusivity and such reduction in thermal diffusivity decreases the temperatures $\theta(\eta)$ and $\phi(\eta)$. Fig. $11(\mathrm{a}, \mathrm{b})$ displays the influence of $\alpha$ on temperature fields $\theta(\eta)$ and $\phi(\eta)$. An increase in $\alpha$ yields a decreases in $\theta(\eta)$ and $\phi(\eta)$. Fig. $12(\mathrm{a}, \mathrm{b})$ describes that $\theta(\eta)$ and $\phi(\eta)$ are decreasing functions of $s$.

Table 1 is prepared to analyze the convergence of $f^{\prime \prime}(0)$, $g^{\prime \prime}(0), \theta^{\prime}(0)$ and $\phi^{\prime \prime}(0)$ numerically. This Table depicts that our series solutions converge from 20th order approximations for velocities and 30th order approximations for PST and PHF. It is also observed that we have to compute less order deformations for velocities in comparison to temperatures $\theta(\eta)$ and $\phi(\eta)$. Table 2 shows the comparison with the previous results for various values of $\alpha$ for $f^{\prime \prime}(0), g^{\prime \prime}(0), f(\infty)$ and $g(\infty)$. It is anticipated that our present results are in an excellent agreement with the previous results. Also we analyzed that the numerical values of $f^{\prime \prime}(0), g^{\prime \prime}(0)$, and $g(\infty)$. are increased. Table 3 shows a comparison of $\theta^{\prime}(0)$ for different values of $\alpha, \gamma$ and $s$. One can see that the provided values in the present analysis have an excellent agreement with the values of existing solutions (Liu and Andersson, 2008).

\section{CONCLUSIONS}

In this study, the three-dimensional flow generated by bidirectional stretching surface is investigated in the presence of prescribed surface temperature (PST) and prescribed heat flux (PHF). Interesting observations are mentioned below:

i. Effects of Deborah numbers $\beta_{1}$ and $\beta_{2}$ are quite opposite on the prescribed temperature $\theta(\eta)$;

ii. The temperatures $\theta(\eta)$ and $\phi(\eta)$ are decreasing functions of Deborah number $\beta_{2}$;

iii. A rise in Prandtl number decrease temperature profiles $\theta(\eta), \phi(\eta)$ and thermal boundary layer thickness;

iv. Increasing $\alpha$ reduces $\theta(\eta)$ and $\phi(\eta)$;

v. Increasing $\alpha$ increases the temperature gradient $\theta^{\prime}(0)$.

Acknowledgement. The useful suggestions of reviewers are greatly appreciated. This paper was funded by the Deanship of Scientific Research (DSR), King Abdulaziz University, Jeddah under grant no. (26-130-35-HiCi). The authors, therefore, acknowledge with thanks DSR technical and financial support. 


\section{REFERENCES}

Ahmad, I., Ahmed, M., Abbas, Z., Sajid, M., 2011. Hydromagnetic flow and heat transfer over a bidirectional stretching surface in a porous medium. Thermal Sci., 15, S205-S220.

Ariel, P.D., 2007. The three-dimensional flow past a stretching sheet and the homotopy perturbation method. Comput. Math. Appl., 54, 920-925.

Bhattacharyya, K., 2012. Mass transfer on a continuous flat plate moving in parallel or reversely to a free stream in the presence of chemical reaction. Int. J. Heat Mass Transfer, 55, 3482-3487.

Crane, L.J., 1970. Flow past a stretching plate. Z. Angew. Math. Phys., 21, 645-647.

Fetecau, C., 2003. The Rayleigh-Stokes problem for an edge in an Oldroyd-B fluid. C. R. Acad. Paris Ser., I 335, 979-984.

Fetecau, C., Fetecau, C., 2005. Decay of potential vortex in an Oldroyd-B fluid. Int. J. Eng. Sci., 43, 340-351.

Fetecau, C., Prasad, S.C., Rajagopal, K.R., 2007. A note on the flow induced by a constantly accelerating plate in an Oldroyd-B fluid. Appl. Math. Model., 31, 647-654.

Harris, J., 1977. Rheology and non-Newtonian flow. Longman, London, United Kingdom.

Hayat, T., Shehzad, S.A., Qasim, M., Obaidat, S., 2011. Thermal radiation effects on the mixed convection stagnation-point flow in a Jeffery fluid. Z. Naturforsch., 66a, 606-614.

Hayat, T., Shehzad, S.A., Mustafa, M., Hendi, A.A., $2012 \mathrm{a}$. MHD flow of an Oldroyd-B fluid thorough a porous channel. Int. J. Chemical Reactor Eng., 10, A8.

Hayat, T., Shehzad, S.A., Qasim, M., Obaidat, S., 2012 b. Radiative flow of a Jeffery fluid in a porous medium with power law heat flux and heat source. Nuclear Eng. Design, 243, 15-19.

Hayat, T., Shehzad, S.A., Alsaedi, A., Alhothuali, M.S., 2012c. Mixed convection stagnation point flow of Casson fluid with convective boundary conditions. Chin. Phys. Lett., 29, 114704.

Hayat, T., Shehzad, S.A., Alsaedi, A., 2012d. Soret and Dufour effects in magnetohydrodynamic (MHD) flow of Casson fluid. Appl. Math. Mech., 33, 1301-1312.

Jamil, M., Fetecau, C., 2010. Some exact solutions for rotating flows of a generalized Burgers' fluid in cylindrical domain. J. Non-Newtonian Fluid Mech., 165, 1700-1712.

Jamil, M., Fetecau, C., 2012. Starting solutions for the motion of a generalized Burgers' fluid between coaxial cylinders. Boundary Value Problems, 2012, 14.

Jamil, M., Khan, N.A., Zafar, A.A., 2011. Translational flows of an Oldroyd-B fluid with fractional derivatives. Comput. Math. Appl., 62, 1540-1553.

Kandasamy, R., Periasamy, K., Prabhu, K.K.S., 2005. Effects of chemical reaction, heat and mass transfer along a wedge with heat source and concentration in the presence of suction or injection. Int. J. Heat Mass Transfer, 48, 1388-1394.

Kandasamy, R., Hayat, T., Obaidat, S., 2011. Group theory transformation for Soret and Dufour effects on free convective heat and mass transfer with thermophoresis and chemical reaction over a porous stretching surface in the presence of heat source/sink. Nuclear Eng. Design, 241, 2155-2161.
Kazem, S., Shaban, M., Abbasbandy, S., 2011. Improved analytical solutions to a stagnation-point flow past a porous stretching sheet with heat generation. J. Franklin Institute, 348, 2044-2058.

Keimanesh, M., Rashidi, M.M., Chamkha, A.J., Jafari, R., 2011. Study of a third grade non-Newtonian fluid flow between two parallel plates using the multi-step differential transform method. Comput. Math. Appl., 62, 2871-2891.

Liao, S.J., 2003. Beyond perturbation: Introduction to homotopy analysis method. Chapman and Hall, CRC Press, Boca Raton.

Liu, I-C., Andersson, H.I., 2008. Heat transfer over a bidirectional stretching sheet with variable thermal conditions. Int. J. Heat Mass Transfer, 51, 4018-4024.

Mukhopadhyay, S., Bhattacharyya, K., Layek, G.C., 2011. Slip effects on boundary layer stagnation point flow and heat transfer towards a shrinking sheet. Int. J. Heat Mass Transfer, 54, 2751-2757.

Qi, H., Jin, H., 2009. Unsteady helical flows of generalized Oldroyd-B fluid with fractioanl derivative. Nonlinear Analysis: Real World Appl., 10, 2700-2708.

Rashidi, M.M., Domairry, G., 2009. New analytical solution of the three-dimensional Navier-Stokes equations. Mod. Phys. Lett. B, 23, 3147.

Rashidi, M.M., Keimanesh, M., 2010. Using differential transform method and Pade approximant for solving MHD flow in a laminar liquid film from a horizontal stretching surface. Math. Problems Engin., 2010, 491319.

Rashidi, M.M., Pour, S.A.M., 2010. Analytic approximate solutions for unsteady boundary-layer flow and heat transfer due to a stretching sheet by homotopy analysis method. Nonlinear Analysis: Modelling and Control, 15, 83-95.

Rashidi, M.M., Pour, S.A.M., Abbasbandy, S., 2011. Analytic approximate solutions for heat transfer of a micropolar fluid through a porous medium with radiation. Commun. Nonlinear Sci. Numer. Simulat., 16, 1874-1889.

Schichting, H., 1964. Boundary Layer Theory. 6th Edition. McGraw-Hill, New York, USA.

Shehzad, S.A., Alsaedi, A., Hayat, T., 2012. Three-dimensional flow of Jeffery fluid with convective surface boundary conditions. Int. J. Heat Mass Transfer, 55, 3971-3976.

Tong, D., Zhang, X., Zhang, X., 2009. Unsteady helical flows of generalized Oldroyd-B fluid. J. Non-Newtonian Fluid Mech., 156, 75-83.

Turkyilmazoglu, M., 2010. A note on the homotopy analysis method. Appl. Math. Lett., 23, 1226-1230.

Vosughi, H., Shivanian, E., Abbasbandy, S., 2011. A new analytical technique to solve Volterra's integral equations. Math. Methods Appl. Sci., 34, 1243-1253.

Wang, C.Y., 1984. The three-dimensional flow due to a stretching sheet. Phys. Fluids, 27, 1915-1917.

Zhang, L., Li, Y., Zhang, X., 2011. Exact solutions for MHD generalized Oldroyd-B fluid due to an infinite accelerating plate. Math. Comput. Modelling, 54, 780-788.

Zheng, L., Liu, Y., Zhang, X., 2012. Slip effects on MHD flow of generalized Oldroyd-B fluid with fractional derivative. Nonlinear Analysis: Real World Appl., 13, 513-523.

Received 19 July 2013 Accepted 31 January 2014 